\title{
Philosophiques
}

Jérôme Dokic et Pascal Engel, Ramsey. Vérité et Succès, Paris, Presses Universitaires de France, collection Philosophies, 2001, 128 pages.

\section{Mathieu Marion}

Volume 31, numéro 1, printemps 2004

Poincaré et la théorie de la connaissance

URI : https://id.erudit.org/iderudit/008950ar

DOI : https://doi.org/10.7202/008950ar

Aller au sommaire du numéro

Éditeur(s)

Société de philosophie du Québec

ISSN

0316-2923 (imprimé)

1492-1391 (numérique)

Découvrir la revue

Citer ce compte rendu

Marion, M. (2004). Compte rendu de [Jérôme Dokic et Pascal Engel, Ramsey.

Vérité et Succès, Paris, Presses Universitaires de France, collection Philosophies, 2001, 128 pages.] Philosophiques, 31(1), 266-269.

https://doi.org/10.7202/008950ar d'utilisation que vous pouvez consulter en ligne.

https://apropos.erudit.org/fr/usagers/politique-dutilisation/ 
thématiques qui y sont adoptées rendent justice à la variété des questions développées dans les $R l$, et surtout à la perspective de recherche de chaque contribution. À ce titre, on s'explique mal pourquoi les textes de Courtine (deuxième partie) et Brisart (troisième partie) ne sont pas regroupés dans une même section, puisqu'ils s'intéressent tous deux à la même question, en l'occurrence celle du sens du dépassement, par Husserl, de la théorie du contenu et de l'objet de Twardowski. De manière similaire, on aurait pu souhaiter que les textes de Benoist (troisième partie) et de Lavigne (cinquième partie) soient mis minimalement en relation, puisqu'ils présentent des arguments pour et contre une interprétation des $R l$ en termes de neutralité métaphysique. Malgré ces détails de structure, on retiendra la valeur et l'originalité des contributions individuelles — et c'est à ce titre qu'il faut aussi voir «La représentation vide » de Husserl - , qui assurent indéniablement la pertinence de ce livre dans le cadre des recherches en phénoménologie.

GUILLAUME FRÉCHETTE

Universität Hamburg

\section{Jérôme Dokic et Pascal Engel, Ramsey. Vérité et Succès, Paris, Presses Universitaires de France, collection Philosophies, 2001, 128 pages.}

Frank Ramsey est mort tragiquement, des suites d'une opération, en janvier 1930. Il n'était âgé que de 26 ans. Pourtant, son œuvre, qui vient à peine de paraître en traduction française, en un volume (F. P. Ramsey, Logique, philosophie et probabilités, P. Engel \& M. Marion (dir.), Paris, Vrin, 2003), est d'une importance remarquable. Il ne publia qu'un article de mathématiques, dans lequel il donna une solution partielle à l'Entscheidungsproblem de Hilbert à l'aide d'une paire de théorèmes de combinatoire qui sont à eux seuls à l'origine d'une branche des mathématiques contemporaines qui porte son nom, la «théorie de Ramsey». Il ne publia que deux articles d'économie afin de répondre à des questions soulevées par des collègues économistes à Cambridge, mais ceux-ci furent aussi à l'origine des branches de la théorie économique contemporaine telles que la théorie des «prix de Ramsey». Le texte posthume "Vérité et probabilité» contient une formulation de la théorie des probabilités subjectives et de la théorie de la décision, dont certains résultats furent redécouverts dans les années cinquante, et qui en fait un des grands classiques de l'histoire de ces deux théories. Bien qu'il n'ait point souffert des tortures existentielles que le commun des mortels associe à ce mot, il mérite le titre de «génie » avant nombre de ses contemporains. Malheureusement, en philosophie, sa réputation a été éclipsée par celle de son contemporain à Cambridge et son ami, Ludwig Wittgenstein, qui reconnaissait pourtant l'influence décisive de Ramsey sur son œuvre. En France, à part quelques-uns - par exemple, G.-G. Granger et J. Bouveresse -, on ignorait tout de lui, jusqu'à son nom. Les jeunes générations, qui ont rejeté les ornières paroissiales de leurs aînés pour vivre au rythme d'une Europe ouverte et multiculturelle, le découvrent enfin grâce à ce court livre de Jérôme Dokic et Pascal Engel, dont une traduction anglaise vient par ailleurs de paraître: Frank Ramsey: Truth and Success (Londres, Routledge, 2003).

L'influence des conceptions de la croyance et de la connaissance, de la causalité, des universaux, de Ramsey sur la philosophie s'est fait sentir au long du 
siècle. J'ai mentionné Wittgenstein, mais les anticipations et parallèles avec Davidson sont parfois saisissants. Par ailleurs, l'idée des "énoncés de Ramsey» (p. 56) fut utilisée de diverses façons par Carnap et David Lewis, le «test de Ramsey» (p. 54) a été repris par Robert Stalnaker et Peter Gärdenfors, etc. Le cœur du présent ouvrage est la "sémantique du succès» (p. 71 sq.), qui trouve son origine dans un texte de Jamie Whyte, "Success Semantics» (Analysis, vol. 50 (1990), p. 149-157). Il s'agit d'une tentative d'explication en termes naturalistes des notions de vérité et d'intentionnalité des attitudes propositionnelles à partir de ce qu'il est convenu d'appeler le "principe de Ramsey»(PR), que Dokic et Engel reformulent ainsi: "Les conditions de vérité d'une croyance sont les conditions réelles qui garantissent le succès de l'action que la croyance est susceptible de produire, quel que soit le désir en jeu»(p. 71). L'ouvrage n'est donc pas une introduction à l'ensemble de l'œuvre de Ramsey mais, du point de vue particulier de cette sémantique du succès, une introduction à ses parties centrales (à l'exclusion surtout des questions de philosophie des mathématiques), c'est-à-dire la théorie pragmatiste de la vérité, de la croyance et de l'action (théorie qui sous-tend ses contributions fondamentales aux disciplines que sont la théorie des probabilités et la théorie de la décision, la théorie des jeux et la théorie du choix rationnel), dans la forme quasi achevée que l'on retrouve dans les écrits de 1926-1928, tels que "Faits et Propositions " et "Vérité et Probabilité ». (Dokic et Engel couvrent cependant d'autres d'aspects moins directement liés à la sémantique du succès, tels que la critique de la distinction entre particuliers et universaux par Ramsey, $c f$. p. 5970.) Cette théorie est basée sur une thèse pragmatique - les rapports avec Peirce sont mis de l'avant tout au long de l'ouvrage - énoncée à la fin de «Faits et propositions", selon laquelle la signification d'un énoncé doit être définie en référence aux actions auxquelles son assertion conduirait (Ramsey, Logique, philosophie et probabilités, op. cit., p. 228). Ramsey prend pour exemple le cas d'un poulet qui croit que les chenilles d'un certain type sont empoisonnées et qui s'abstient donc d'en manger, pour cause de maux d'estomacs. Pour Ramsey, tout ensemble d'actions dont l'utilité $p$ est une condition nécessaire et suffisante est appelé une croyance que $p$, et la croyance que $p$ est vraie si $p$, c'est-à-dire si elle est utile. Ramsey écrivait en effet: "On pourrait très bien soutenir que relativement à ce type de croyance la théorie pragmatiste est correcte, c'est-à-dire que la relation entre le comportement du poulet et les facteurs objectifs était que les actions seraient de nature telles qu'elles seraient utiles si, et seulement si, les chenilles étaient empoisonnées. Ainsi, n'importe quelle sorte d'action pour l'utilité desquelles $p$ est une condition nécessaire et suffisante pourrait être appelée une croyance que $p$, et serait vraie si $p$, c'est-à-dire si elles sont utiles» (Ramsey, Logique, philosophie et probabilités, op. cit., p. 218). On voit à cela que, selon Ramsey, une croyance est une sorte de "guide" pour nos actions, et une croyance est vraie si elle conduit à une action bénéfique, c'est-à-dire si elle est utile. Dans l'exemple de Ramsey, la croyance que la chenille est empoisonnée conduit à l'action de s'abstenir de la manger, et cette croyance est vraie si elle est utile, c'est-à-dire si la chenille est en effet empoisonnée et le poulet évite donc des maux d'estomac. De la même manière, l'action de manger la chenille indique la croyance que la chenille est mangeable, et cette croyance serait fausse si le poulet se retrouvait avec des maux d'estomac. Il s'agit ici du cas des croyances «pleines » (c'est-à-dire de probabilité 0 ou 1); Ramsey offre aussi une explication très intéressante dans le cas des croyances 
partielles. Si le poulet ne sait pas si la chenille est empoisonnée ou pas, il devrait agir de manière à maximiser (tout en tenant compte des fréquences objectives) son utilité subjective. Mais comment mesurer celle-ci? Étant donné que les agents révèlent leurs préférences dans leur conduite, Ramsey propose de déterminer les degrés de probabilité subjective et les degrés d'utilité des agents par une méthode de paris. Les croyances conditionnelles les mieux justifiées sont donc celles qui ont les meilleures conditions de succès (et qui sont corrélées à la fréquence objective la plus élevée). D’où le principe de Ramsey, selon lequel «Les croyances ont d'autant plus de chances d'être vraies que leurs conditions de succès sont garanties» (p. 27). Cependant, comme Dokic et Engel l'ont très bien vu (p. 31-41), Ramsey ne cherchait pas à donner une définition pragmatique de la vérité, mais une explication du contenu des croyances en termes de conditions de vérité, d'où la reformulation du PR (p. 71) déjà citée.

Les deux premiers chapitres de l'ouvrage constituent une introduction détaillée à cette théorie de Ramsey, pour motiver l'idée d'une sémantique du succès présentée dans le troisième chapitre; les deux derniers abordent chacun une objection majeure à la sémantique du succès. L'avant-dernier chapitre porte sur le problème de la satisfaction des désirs, posé en ces termes: "Selon le pragmatisme ramseyen, les conditions de vérité des croyances sont celles qui garantissent la réussite de l'action. Or une action est réussie lorsque le désir qui la motive est satisfait. PR revient donc à définir les conditions de vérité des croyances par référence aux conditions de satisfaction des désirs. Mais peut-on déterminer les secondes indépendamment des premières?»(p. 84). Dans le dernier chapitre, «La situation de l'action" (p. 100-122), Dokic et Engel discutent l'objection "de la surcharge cognitive " (cf., par exemple, R. Brandom, "Unsuccessful Semantics ", Analysis, 54, 1994, 175-178.) En effet, le PR implique que l'échec d'une action a doit être rapporté à une croyance fausse ayant joué un rôle dans la décision de faire a. Cela suppose cependant une omniscience de l'agent, qui doit entretenir des croyances pour toutes les circonstances pertinentes pour la réussite de l'action; cette condition n'est pas du tout réaliste et les contre-exemples abondent où l'échec est dû non pas à une croyance fausse mais à l'ignorance.

Nonobstant la réplique adroite à cette objection de Dokic et Engel, j'aimerais pointer du doigt ce qui me semble être un défaut dans leur lecture de Ramsey. Dans la dernière année de sa vie (1929), Ramsey a remis en cause un certain nombre de ses vues. Sa mort subite l'a empêché de développer ses nouvelles idées, dont il ne reste que des écrits posthumes, certes nombreux, mais qui ne recomposent pas une théorie achevée comme celle de 1926-1928, qui sert de point de départ à Dokic et Engel. Ceux-ci connaissent, bien sûr, ces ultimes développements de la pensée de Ramsey ( $c f$., par exemple, la présentation du point de vue sur les croyances générales, p. 48 sq.), mais à mon avis ils ne tiennent pas suffisamment compte du fait que les nouvelles idées vont à l'encontre de certaines des idées essentielles de la théorie de 1926-1928. En un mot, pour Ramsey, les croyances sont, telles une carte, des "guides" pour l'action, mais une carte infinie n'est pas manipulable et ne peut pas être un guide pour l'action ( $c f$. Ramsey, Logique, philosophie et probabilités, op. cit., p. 238). Les croyances générales doivent donc être conçues autrement que comme des propositions infinies, d'où l'introduction, en 1929, de la notion d' "hypothétique avec variable» (variable hypothetical), qui le mena à l'adoption d'un point de vue finitiste en mathématiques et en philosophie des 
sciences (cf. Ramsey, Logique, philosophie et probabilités, op. cit., p. 252). Nous sommes certes loin des préoccupations actuelles quant à la question de la complexité. Mais il est clair que la théorie de 1926-1928 de Ramsey, tout comme la récente "sémantique du succès ", a été élaborée sans prendre en considération ces problèmes; elles participent, si l'on veut, du paradigme de la rationalité «instrumentale». Ramsey avait lui-même ouvert la porte à la remise en cause des idéalisations fortes (telles que l'omniscience dénoncée par l'objection de la surcharge cognitive ou, pour ne prendre qu'un autre exemple, la révision des croyances par "conditionalisation» — qui n'a cependant pas de lien avec le soidisant «test de Ramsey» - , qui est une fonction à croissance exponentielle et qui n'est donc pas «faisable» en pratique) que comportent sa théorie de 1926-1928 et celles qui s'en inspirent; idéalisations qui expliquent en partie les difficultés rencontrées lorsqu'on veut les appliquer à des cas concrets. N'écrivait-il pas dès "Vérité et probabilité»: "On pourrait dire qu'on devrait penser ce qui est vrai, mais en ce sens là [...] on ne peut non plus trouver quelque justification de la croyance partielle; la chose idéale serait d'avoir des croyances de degré 1 en toute proposition vraie et de degré 0 en toute proposition fausse. Mais c'est trop demander à des hommes mortels, et on doit convenir qu'un certain degré de doute, ou même d'erreur, est humainement justifié» (Ramsey, Logique, philosophie et probabilités, op. cit., p. 176). On est donc en droit d'espérer que ces dernières idées sauront inspirer de nouveaux développements. En attendant, il faut remercier les auteurs pour avoir mis à notre disposition une introduction non seulement à l'œuvre de Ramsey mais aussi à une théorie contemporaine qui en revendique l'héritage.

MATHIEU MARION

Université du Québec à Montréal

\section{Michael Friedman, Dynamics of Reason, Stanford, CSLI Publications, 2001, 141 pages.}

Ce recueil est constitué des conférences sur Kant que l'auteur a prononcées en 1994 à l'Université Stanford dans le cadre d'un cycle de Kant Lectures. Il s'agit moins d'un ensemble d'études sur Kant que d'une tentative de récupérer l'héritage kantien et de le confronter au legs du positivisme logique. Le premier chapitre est en effet consacré à l'idée d'une philosophie scientifique, idée chère à un Hans Reichenbach. Mais c'est surtout l'empirisme logique de Carnap que Friedman veut sauvegarder face au naturalisme prôné par un Quine qui n'aurait pas réussi à prendre la mesure de l'entreprise carnapienne. C'est aussi à un Kuhn reconduit à ses sources rationalistes et contre le relativisme post-kuhnien que nous convie Friedman, qui voudrait dynamiser et en même temps historiciser l'a priori kantien.

L'auteur connaît bien Kant et ses sources scientifiques; son Kant and the Exact Sciences (1992) montre comment Kant se serait laissé entraîner sur la pente glissante de la déduction transcendantale d'une physique (et d'une chimie) fondée sur des principes a priori. L'Opus Postumum esquisse une théorie du "Wärmestoff" ou éther calorique qui voudrait faire le pont entre le transcendantal et l'empirique. On sait que Hegel a voulu remonter cette pente dans une physico-chimie dénaturée, 